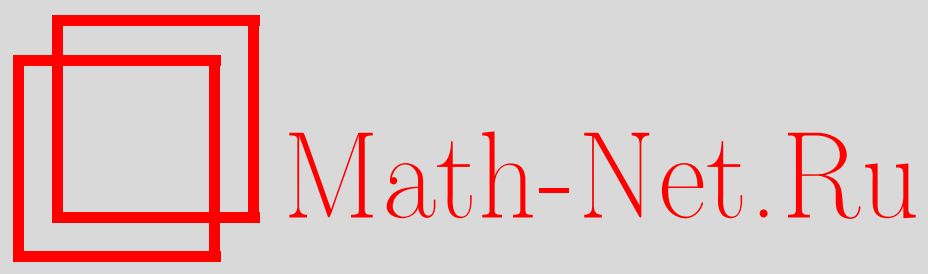

M.

Ю.

Игнатьев,

$\mathrm{O}$

подобии

вольтерровых операторов и операторах преобразования для интегро-дифференциальных уравнений дробных порядков, Матем. заметки, 2003, том 73, выпуск 2, 206-216

DOI: https://doi.org/10.4213/mzm177

Использование Общероссийского математического портала Math-Net.Ru подразумевает, что вы прочитали и согласны с пользовательским соглашением http://www.mathnet.ru/rus/agreement

Параметры загрузки:

IP : 54.166 .219 .16

26 апреля 2023 г., 12:58:27

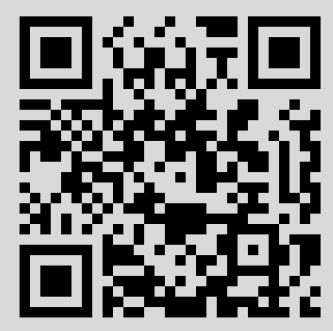




\section{О ПОДОБИИ ВОЛЬТЕРРОВЫХ ОПЕРАТОРОВ \\ И ОПЕРАТОРАХ ПРЕОБРАЗОВАНИЯ \\ ДЛЯ ИНТЕГРО-ДИФ ФЕРЕНЦИАЛЬНЫХ \\ УРАВНЕНИЙ ДРОБНЫХ ПОРЯДКОВ}

\section{М. Ю. Игнатьев}

Устанавливается подобие некоторых интегральных вольтерровых операторов оператору дробного интегрирования Римана-Лиувилля, а также существование треугольного оператора преобразования для интегро-дифференциальных уравнений дробных порядков. Полученные результаты сохраняют преемственность с аналогичными результатами для случая целых порядков.

Библиограффия: 7 названий.

В работе рассматриваются вопросы подобия вольтерровых операторов оператору дробного интегрирования Римана-Лиувилля, а также тесно связанные с ними вопросы существования классического треугольного оператора преобразования для интегро-дифференциальных уравнений дробных порядков. Рассмотрим для вещественных $\alpha>2$ интегральный вольтерров оператор, допускающий представление вида

$$
M=J^{\alpha}+J^{\alpha+1} N,
$$

где $N$ - интегральный вольтерров оператор, $J^{\alpha}-$ оператор дробного интегрирования Римана-Лиувилля

$$
J^{\alpha} f(x)=\int_{0}^{x} \frac{(x-t)^{\alpha-1}}{\Gamma(\alpha)} f(t) d t .
$$

Также рассмотрим следуюший интегро-дифференциальный оператор дробного порядка с параметрами $\alpha=n-\epsilon, n \geqslant 3, \epsilon \in(0,1)$ :

$$
\ell y=D^{\alpha} y+\sum_{k=2}^{n} p_{k}(x) D^{\alpha-k} y+P J^{\epsilon} y
$$

где $P$ - интегральный вольтерров оператор.

Вопросам подобия вольтерровых операторов целым степеням оператора интегрирования посвящено большое количество работ различных авторов; также достаточнополно изучен вопрос о существовании оператора преобразования для дифференциальных и

Работа выполнена при поддержке Российского фонда фундаментальных исследований, грант № 00-01-00741. 
интегро-дифференциальных операторов целых порядков ([1]-[4] и др.). Отметим, что в случае дифференциальных операторов высших порядков $(n>2)$ существенным при построении треугольного оператора преобразования является требование аналитичности коэффициентов [5] (соответственно, для подобия вольтеррова оператора $n$-й степени оператора интегрирования при $n>2$ сушественно требование аналитичности ядра). Случаю нецелых $\alpha$ посвящена работа Маламуда [6].

В настоящей работе используется развитие метода, предложенного в работах [1], [3], [4] и являющегося, в свою очередь, обобщением метода, применявшегося В. А. Марченко при построении оператора преобразования для решений уравнения Штурма-Лиувилля. Предлагаемьй метод позволяет ослабить требования, налагавшиеся в [6] на область аналитичности ядра оператора $M$ и коэффициентов оператора (3), и добиться преемственности с результатами, полученными в [3], [4] для случая целых порядков $\alpha=n \in N$.

Обозначим через $D_{a}$ четырехугольник в комплексной плоскости с вершинами $\{0, a$, $\left.a(1-\omega)^{-1}, a\left(1-\omega^{-1}\right)^{-1}\right\}, \omega=\exp (2 \pi i / \alpha), V=\left\{(x, \xi): x \in[0,1], \xi \in \bar{D}_{1-x}\right\}$. Будем говорить, что интегральньй вольтерров оператор $N$ является оператором класса $A$, если он представим в виде

$$
N f(x)=\int_{0}^{x} N(x-t, t) f(t) d t
$$

где функция $N(x, \xi)$ определена и непрерьвна на $V$ и для любого фиксированного $x \in$ $[0,1]$ как функция $\xi$ аналитична в области $D_{1-x}$.

Теорема 1. Пусть $M$ - оператор вида (1), (2), $N$ - вольтерров оператор класса $A$. Тогда существует вольтерров оператор $K$ класса $A$ такой, что $M=$ $(E+K) J^{\alpha}(E+K)^{-1}$ в пространствах $C[0,1], L_{p}[0,1], p \geqslant 1$.

Пусть функция $y(x, \lambda)$ - решение следуюшей задачи Коши:

$$
\begin{gathered}
\ell y=\lambda y, \\
\left.D^{\alpha-k} y\right|_{x=0}=\delta_{k, n}, \quad k=1, \ldots, n,
\end{gathered}
$$

где $\ell$ - оператор вида $(3), \tilde{y}(x, \lambda)$ - решение “простейшей” задачи Коши:

$$
\begin{gathered}
D^{\alpha} \tilde{y}=\lambda \tilde{y}, \\
\left.D^{\alpha-k} \tilde{y}\right|_{x=0}=\delta_{k, n}, \quad k=1, \ldots, n .
\end{gathered}
$$

Теорема 2. Пусть коэффичиенты интегро-дифференииального оператора (3) аналитичны в $D_{1}$, функиии $p_{k}^{(n-k)}(\xi), k=2, \ldots, n$, непрерывны в $\bar{D}_{1}, P-$ вольтерров оператор класса $A$. Тогда для $y(x, \lambda)$ справедливо представление

$$
y(x, \lambda)=(E+K) \tilde{y}(x, \lambda)=\tilde{y}(x, \lambda)+\int_{0}^{x} K(x-t, t) \tilde{y}(t, \lambda) d t,
$$

әде $K$ - вольтерров оператор класса $A$.

Прежде чем приступать к доказательству теорем 1,2 , установим ряд вспомогательных утверждений. Всюду далее $\alpha \notin \mathbb{N} ; m:=[\alpha / 2]$.

Введем в рассмотрение функцию

$$
\varphi(z, \lambda)=\sum_{n=0}^{\infty} \frac{z^{n \alpha} \lambda^{n}}{\Gamma(n \alpha+1)}
$$

(здесь и далее $\left.z^{\alpha}=|z|^{\alpha} \exp (i \alpha \arg z), \arg z \in(-\pi, \pi]\right)$. 
ЛЕмма 1. Функиия $\varphi(z, \lambda)$ обладает следующими свойствами:

1) $п р u x>0$

$$
\varphi(x, \lambda)=\left(E-\lambda J^{\alpha}\right)^{-1} 1
$$

2) для любих $x>0, j=-m, \ldots, m$

$$
\varphi\left(x \omega^{j}, \lambda\right)=\varphi(x, \lambda)
$$

3) для любих $x>0, y>0$

$\varphi(x, \lambda) \varphi(y, \lambda)=\frac{1}{\alpha} \sum_{j=-m}^{m} \varphi\left(x+\omega^{j} y, \lambda\right)+\int_{0}^{x} g(x-t, y) \varphi(t, \lambda) d t+\int_{0}^{y} g(y-t, x) \varphi(t, \lambda) d t$ əəe

$$
g(x, y)=-\frac{\sin \alpha \pi}{\pi} \frac{x^{\alpha-1} y^{\alpha}}{x^{2 \alpha}-2 x^{\alpha} y^{\alpha} \cos \alpha \pi+y^{2 \alpha}}
$$

ДокАЗАтЕЛЬСтво. Свойства 1), 2) следуют непосредственно из определения (10) функции $\varphi(x, \lambda)$. Докажем свойство 3$)$. Заметим, прежде всего, что обе части равенства (13) - целые функции по $\lambda$, поэтому достаточно установить его справедливость для положительных $\lambda$.

Для доказательства равенства (13) воспользуемся аппаратом двумерного преобразования Лапласа. Условимся, что запись

$$
f(x, y) \stackrel{x, y}{\div} F(p, q)
$$

означает, что функция $F(p, q)$ является двумерным преобразованием Лапласа функции $f(x, y)$ :

$$
F(p, q)=\int_{0}^{+\infty} \int_{0}^{+\infty} f(x, y) \exp (-p x-q y) d x d y
$$

записи

$$
f(x, y) \stackrel{x}{\div} F(p, y), \quad f(x, y) \stackrel{y}{\div} F(x, q)
$$

означают соответственно

$$
F(p, y)=\int_{0}^{+\infty} f(x, y) \exp (-p x) d x, \quad F(x, q)=\int_{0}^{+\infty} f(x, y) \exp (-q y) d y .
$$

С учетом известных теорем единственности для преобразования Лапласа (см., например, $[7$, c. 22]) достаточно доказать равенство двумерных изображений правой и левой частей (13) для $p>\lambda^{1 / \alpha}, q>\lambda^{1 / \alpha}, p \neq q$.

Итак, всюду далее, если не оговорено противное, предполагаем, что $\lambda>0, p>\lambda^{1 / \alpha}$, $q>\lambda^{1 / \alpha}, p \neq q$.

Перейдем к подсчету изображений правой и левой частей (13).

1. Хорошо известно, что $|\varphi(z, \lambda)|<C \exp \left(\lambda^{1 / \alpha}|z|\right), \lambda>0$, и при $\operatorname{Re} p>\lambda^{1 / \alpha}$

$$
\int_{0}^{+\infty} \varphi(x, \lambda) \exp (-p x) d x=\frac{p^{\alpha-1}}{p^{\alpha}-\lambda}
$$


откуда

$$
\varphi(x, \lambda) \varphi(y, \lambda) \stackrel{x, y}{\div} \frac{p^{\alpha-1}}{p^{\alpha}-\lambda} \frac{q^{\alpha-1}}{q^{\alpha}-\lambda} .
$$

Далее, из $[7$, формула $(2.36)$, с. 58$]$

$$
\varphi(x+s y, \lambda) \stackrel{x, y}{\div} \frac{1}{s p-q}\left(\frac{s q^{\alpha-1}}{q^{\alpha}-\lambda s^{\alpha}}-\frac{p^{\alpha-1}}{p^{\alpha}-\lambda}\right), \quad s>0 .
$$

Зафиксируем $p, q$ такие, что $p>\lambda^{1 / \alpha}, q>\lambda^{1 / \alpha}, p \neq q$. Тогда правая часть равенства (17) - аналитическая функция по $s$ в некоторой (односвязной) области вида

$$
\{|\arg s|<\pi,|s| \in(1-\delta, 1+\delta)\}
$$

$\mathrm{C}$ другой стороны, в силу оценки $|\varphi(z, \lambda)|<C \exp \left(\lambda^{1 / \alpha}|z|\right), \lambda>0$, интеграл

$$
\int_{0}^{+\infty} \int_{0}^{+\infty} \varphi(x+s y, \lambda) \exp (-p x-q y) d x d y
$$

представляет собой аналитическую по $s$ функцию в области $\left\{|\arg s|<\pi,|s|<q \lambda^{-1 / \alpha}\right\}$. Поэтому равенство (17) для любых фиксированных $\lambda>0, p>\lambda^{1 / \alpha}, q>\lambda^{1 / \alpha}, p \neq q$ справедливо в некоторой области $\{|\arg s|<\pi,|s| \in(1-\delta, 1+\delta)\}$, в частности, при $s=\omega^{j}, j=-m, \ldots, m$. Таким образом,

$$
\varphi\left(x+\omega^{j} y, \lambda\right) \stackrel{x, y}{\div} \frac{1}{p-q \omega^{-j}} \frac{q^{\alpha-1}}{q^{\alpha}-\lambda}-\frac{1}{p \omega^{j}-q} \frac{p^{\alpha-1}}{p^{\alpha}-\lambda} .
$$

2. Введем функцию

$$
\varphi_{1}(x, \rho)=\varphi\left(x, \rho^{\alpha}\right)-\frac{1}{\alpha} \sum_{j=-m}^{m} \exp \left(\rho \omega^{j} x\right) .
$$

Применяя формулу обращения преобразования Лапласа, получим из (15) после смены контура интегрирования и применения теоремы о вычетах:

$$
\varphi\left(x, \rho^{\alpha}\right)=\frac{1}{2 \pi i} \int_{C^{\star}} \frac{p^{\alpha-1}}{p^{\alpha}-\rho^{\alpha}} \exp (p x) d p+\frac{1}{\alpha} \sum_{j=-m}^{m} \exp \left(\rho \omega^{j} x\right),
$$

где

$$
\begin{aligned}
C^{\star}= & \{p=t \exp (-i \pi), t \in(+\infty, \epsilon]\} \cup\{p=\epsilon \exp (i \gamma), \gamma \in(-\pi, \pi)\} \\
& \cup\{p=t \exp (i \pi), t \in[\epsilon,+\infty)\}
\end{aligned}
$$

Откуда

$$
\begin{aligned}
\varphi_{1}(x, \rho) & =\frac{1}{2 \pi i} \int_{C^{\star}} \frac{p^{\alpha-1}}{p^{\alpha}-\rho^{\alpha}} \exp (p x) d p=\frac{1}{2 \pi i} \int_{C^{\star}} \frac{s^{\alpha-1}}{s^{\alpha}-1} \exp (\rho s x) d s \\
& =\left.\int_{0}^{+\infty}\left(\frac{1}{2 \pi i} \frac{s^{\alpha-1}}{s^{\alpha}-1}\right)\right|_{s=\tau \exp (i \pi)} ^{s=\tau \exp (-i \pi)} \exp (-\rho \tau x) d \tau .
\end{aligned}
$$


После подсчета разности значений на верхнем и нижнем берегах разреза получим

$$
\varphi_{1}(x, \rho)=-\frac{\sin \alpha \pi}{\pi} \int_{0}^{+\infty} \frac{\tau^{\alpha-1}}{\tau^{2 \alpha}-2 \tau^{\alpha} \cos (\alpha \pi)+1} \exp (-\rho \tau x) d \tau .
$$

Наконец, произведя замену переменной $\tau x=t$, получим окончательно

$$
\varphi_{1}(x, \rho)=\int_{0}^{+\infty} g(t, x) \exp (-\rho t) d t
$$

где $g(t, x)$ - функция, определяемая равенством (14). Далее, из (19), в частности, следует, что интеграл Лапласа (по $x$ ) для $\varphi_{1}(x, \rho)$ сходится абсолютно при любом $p>0$, и, таким образом, с учетом (15) для любого $p>0$ имеем

$$
\int_{0}^{+\infty} \varphi_{1}(x, \rho) \exp (-p x) d x=\frac{p^{\alpha-1}}{p^{\alpha}-\rho^{\alpha}}-\frac{1}{\alpha} \sum_{j=-m}^{m} \frac{1}{p-\rho \omega^{j}} .
$$

Из (19), (20) имеем

$$
\begin{gathered}
g(x, y) \stackrel{x}{\div} \varphi_{1}(y, p), \quad p>0, \\
\varphi_{1}(y, p) \stackrel{y}{\div} \frac{q^{\alpha-1}}{q^{\alpha}-p^{\alpha}}-\frac{1}{\alpha} \sum_{j=-m}^{m} \frac{1}{q-p \omega^{j}}, \quad p>0, \quad q>0
\end{gathered}
$$

откуда

$$
g(x, y) \stackrel{x, y}{\div} \frac{q^{\alpha-1}}{q^{\alpha}-p^{\alpha}}-\frac{1}{\alpha} \sum_{j=-m}^{m} \frac{1}{q-p \omega^{j}}, \quad p>0, \quad q>0 .
$$

3) Согласно теореме о свертке [7, теорема 13, с. 37]

$$
\begin{aligned}
& \int_{0}^{x} g(x-t, y) \varphi(t, \lambda) d t+\int_{0}^{y} g(y-t, x) \varphi(t, \lambda) d t \\
& \quad \stackrel{x, y}{\div}\left(\frac{q^{\alpha-1}}{q^{\alpha}-p^{\alpha}}-\frac{1}{\alpha} \sum_{j=-m}^{m} \frac{1}{q-p \omega^{j}}\right) \frac{p^{\alpha-1}}{p^{\alpha}-\lambda}+\left(\frac{p^{\alpha-1}}{p^{\alpha}-q^{\alpha}}-\frac{1}{\alpha} \sum_{j=-m}^{m} \frac{1}{p-q \omega^{j}}\right) \frac{q^{\alpha-1}}{q^{\alpha}-\lambda} .
\end{aligned}
$$

Утверждение леммы следует теперь непосредственно из (16), (18), (21). Лемма доказана.

Рассмотрим, далее, оператор

$$
\Phi_{\lambda} f(x):=\int_{0}^{x} \varphi(x-t, \lambda) f(t) d t .
$$


ЛЕмма 2. Пусть для функиии $f(x, \lambda)$ справедливо представление

$$
f(x, \lambda)=F \varphi(x, \lambda)=\int_{0}^{x} F(x-t, t) \varphi(t, \lambda) d t
$$

где $F$ - вольтерров оператор класса $A$. Тогда для функиии $h=\Phi_{\lambda} f$ справедливо представление

$$
h(x, \lambda)=H \varphi(x, \lambda)=\int_{0}^{x} H(x-t, t) \varphi(t, \lambda) d t,
$$

где $H$ - вольтерров оператор класса $A$ я ядром

$$
\begin{gathered}
H(x, \xi)=\widetilde{H}(x)+\widehat{H}(x, \xi)+\sum_{j=-m}^{m} H_{j}(x, \xi), \\
\widetilde{H}(x)=\int_{0}^{x} d t \int_{0}^{t} g(x-t, t-\tau) F(\tau, t-\tau) d \tau, \\
\widehat{H}(x, \xi)=\int_{0}^{x} d t \int_{0}^{t} g(t-\tau, x-t) F(\tau, t-\tau+\xi) d \tau, \\
\alpha H_{j}(x, \xi)=\frac{\omega^{j}}{1-\omega^{j}} \int_{0}^{x} F\left(\tau, \xi+\frac{x-\tau}{1-\omega^{j}}\right) d \tau-\frac{1}{1-\omega^{j}} \int_{0}^{x} F\left(\tau, \frac{x-\tau}{1-\omega^{j}}\right) d \tau, \\
\alpha H_{0}(x, \xi)=\int_{0}^{\xi} F(x, t) d t .
\end{gathered}
$$

ДокАЗАТЕЛЬСТво. Имеем

$$
\begin{aligned}
h(x, \lambda) & =\int_{0}^{x} \varphi(x-t, \lambda) d t \int_{0}^{t} F(t-\tau, \tau) \varphi(\tau, \lambda) d \tau \\
& =\int_{0}^{x} \int_{0}^{t} F(\tau, t-\tau) \varphi(x-t, \lambda) \varphi(t-\tau, \lambda) d \tau d t .
\end{aligned}
$$

Применяя лемму 1 , получим

$$
\begin{gathered}
h(x, \lambda)=\tilde{h}(x, \lambda)+\hat{h}(x, \lambda)+\frac{1}{\alpha} \sum_{j=-m}^{m} h_{j}(x, \lambda), \\
\tilde{h}(x, \lambda)=\int_{0}^{x} d t \int_{0}^{t} d \tau \int_{0}^{x-t} F(\tau, t-\tau) g(x-t-\xi, t-\tau) \varphi(\xi, \lambda) d \xi, \\
\hat{h}(x, \lambda)=\int_{0}^{x} d t \int_{0}^{t} d \tau \int_{0}^{t-\tau} F(\tau, t-\tau) g(t-\tau-\xi, x-t) \varphi(\xi, \lambda) d \xi, \\
h_{j}(x, \lambda)=\int_{0}^{x} d t \int_{0}^{t} F(\tau, t-\tau) \varphi\left(x-t+\omega^{j}(t-\tau), \lambda\right) d \tau .
\end{gathered}
$$

Из (29) после замены переменной $t \rightarrow x-t$ и смены порядка интегрирования получим

$$
\begin{aligned}
\tilde{h}(x, \lambda) & =\int_{0}^{x} \varphi(\xi, \lambda) d \xi \int_{\xi}^{x} d t \int_{0}^{x-t} F(\tau, x-t-\tau) g(t-\xi, x-t-\tau) d \tau \\
& =\int_{0}^{x} \widetilde{H}_{0}(x, \xi) \varphi(\xi, \lambda) d \xi
\end{aligned}
$$


причем

$$
\begin{aligned}
\widetilde{H}(x) & :=\widetilde{H}_{0}(x+\xi, \xi)=\int_{\xi}^{\xi+x} d t \int_{0}^{\xi+x-t} F(\tau, x+\xi-t-\tau) g(t-\xi, x+\xi-t-\tau) d \tau \\
& =\int_{0}^{x} d t \int_{0}^{t} g(x-t, t-\tau) F(\tau, t-\tau) d \tau
\end{aligned}
$$

Аналогично из (30) после замены $\tau \rightarrow t-\tau$ и смены порядка интегрирования получаем

$$
\begin{aligned}
\hat{h}(x, \lambda)= & \int_{0}^{x} \varphi(\xi, \lambda) d \xi \int_{\xi}^{x} d t \int_{\xi}^{t} F(t-\tau, \tau) g(\tau-\xi, x-t) d \tau=\int_{0}^{x} \widehat{H}_{0}(x, \xi) \varphi(\xi, \lambda) d \xi \\
\widehat{H}(x, \xi) & :=\widehat{H}_{0}(x+\xi, \xi)=\int_{\xi}^{\xi+x} d t \int_{\xi}^{t} F(t-\tau, \tau) g(\tau-\xi, x+\xi-t) d \tau \\
& =\int_{0}^{x} d t \int_{\xi}^{\xi+x} F(t-\tau+\xi, \tau) g(\tau-\xi, x-t) d \tau \\
& =\int_{0}^{x} d t \int_{0}^{t} F(\tau, t-\tau+\xi) g(t-\tau, x-t) d \tau .
\end{aligned}
$$

Далее, из (31) при $j \neq 0$ после смены порядка интегрирования и замен переменных $t \rightarrow \xi=x-t+\omega^{j}(t-\tau), \tau \rightarrow x-\tau$ получим

$$
h_{j}(x, \lambda)=\frac{1}{1-\omega^{j}} \int_{0}^{x} d \tau \int_{\tau}^{\omega^{j} \tau} F\left(x-\tau, \frac{\tau-\xi}{1-\omega^{j}}\right) \varphi(\xi, \lambda) d \xi .
$$

В силу аналитических свойств функции $F(x, \xi)$ подьптегральная функция во внутреннем интеграле аналитична в треугольнике с вершинами $\left\{0, \tau, \omega^{j} \tau\right\}$ и непрерьвна в его замькании. Поэтому

$$
\begin{aligned}
h_{j}(x, \lambda)= & \frac{1}{1-\omega^{j}} \int_{0}^{x} d \tau \int_{0}^{\omega^{j} \tau} F\left(x-\tau, \frac{\tau-s}{1-\omega^{j}}\right) \varphi(s, \lambda) d s \\
& -\frac{1}{1-\omega^{j}} \int_{0}^{x} d \tau \int_{0}^{\tau} F\left(x-\tau, \frac{\tau-\xi}{1-\omega^{j}}\right) \varphi(\xi, \lambda) d s .
\end{aligned}
$$

Вьполнив в первом интеграле замену $s=\xi \omega^{j}$ и воспользовавшись $(12)$, получим

$$
\begin{gathered}
h_{j}(x, \lambda)=\int_{0}^{x} \widetilde{H}_{j}(x, \xi) \varphi(\xi, \lambda) d \xi \\
\widetilde{H}_{j}(x, \xi)=\frac{\omega^{j}}{1-\omega^{j}} \int_{\xi}^{x} F\left(x-\tau, \frac{\tau-\xi \omega^{j}}{1-\omega^{j}}\right) d \tau-\frac{1}{1-\omega^{j}} \int_{\xi}^{x} F\left(x-\tau, \frac{\tau-\xi}{1-\omega^{j}}\right) d \tau .
\end{gathered}
$$

Далее,

$$
\begin{aligned}
\alpha H_{j}(x, \xi):= & \widetilde{H}_{j}(x+\xi, \xi) \\
= & \frac{\omega^{j}}{1-\omega^{j}} \int_{\xi}^{x+\xi} F\left(x+\xi-\tau, \frac{\tau-\xi \omega^{j}}{1-\omega^{j}}\right) d \tau \\
& -\frac{1}{1-\omega^{j}} \int_{\xi}^{x+\xi} F\left(x+\xi-\tau, \frac{\tau-\xi}{1-\omega^{j}}\right) d \tau \\
= & \frac{\omega^{j}}{1-\omega^{j}} \int_{0}^{x} F\left(\tau, \xi+\frac{x-\tau}{1-\omega^{j}}\right) d \tau-\frac{1}{1-\omega^{j}} \int_{0}^{x} F\left(\tau, \frac{x-\tau}{1-\omega^{j}}\right) d \tau .
\end{aligned}
$$


Наконец, непосредственным подсчетом легко проверяется, что

$$
h_{0}(x, \lambda)=\int_{0}^{x} H_{0}(x-\xi, \xi) \varphi(\xi, \lambda) d \xi, \quad H_{0}(x, \xi)=\int_{0}^{x} F(x, t) d t
$$

Указанное в лемме представление получается теперь объединением равенств (28)-(39). Требуемые аналитические свойства функций $H_{j}(x, \xi)$ устанавливаются аналогично [2], аналитические свойства функции $\widehat{H}(x, \xi)$ следуют из аналитических свойств функции $F(x, \xi)$, представления $(25)$ и очевидного включения $t-\tau+D_{1-t} \subseteq D_{1-\tau}, \tau \in[0, t]$. Лемма доказана.

Следующее утверждение легко проверить непосредственным подсчетом.

Лемма 3. Пусть $N_{1}, N_{2}$ - вольтерровы операторы класса A. Тогда операmор $N_{2,1}=N_{2} N_{1}-$ maкже оператор класса $A$ :

$$
N_{2,1} f(x)=\int_{0}^{x} N_{2,1}(x-t, t) f(t) d t
$$

$2 \partial e$

$$
N_{2,1}(x, \xi)=\int_{0}^{x} N_{2}(x-\tau, \xi+\tau) N_{1}(\tau, \xi) d \tau .
$$

Далее, если $N-$ вольтерров оператор класса $A$, mо $(E+N)^{-1}=E+N_{1}$, дде $N_{1}-$ также вольтерров оператор класса $A$.

ДоКАЗАТЕЛЬСТВо ТЕОРЕМЫ 1. Введем в рассмотрение функцию

$$
y(x, \lambda)=(E-\lambda M)^{-1} 1
$$

Используя (1), (11), и учитьвая, что, как следует из (22),

$$
J+\lambda\left(E-\lambda J^{\alpha}\right)^{-1} J^{\alpha+1}=J\left(E-\lambda J^{\alpha}\right)^{-1}=\Phi_{\lambda}
$$

получим следующее представление:

$$
y=\left(E+\left(J-\Phi_{\lambda}\right) N\right)^{-1} \varphi=(E+J N)^{-1}\left(E-\Phi_{\lambda} N(E+J N)^{-1}\right)^{-1} \varphi
$$

Определим

$$
y_{1}(x, \lambda):=(E+J N) y(x, \lambda), \quad N_{1}:=N(E+J N)^{-1} .
$$

В силу леммы $3 N_{1}$ - интегральньй вольтерров оператор класса $A$. Далее имеем

$$
y_{1}(x, \lambda)=\left(E-\Phi_{\lambda} N_{1}\right)^{-1} \varphi(x, \lambda)=\varphi(x, \lambda)+\sum_{n=1}^{\infty} \varphi_{n}(x, \lambda)
$$

где

$$
\varphi_{1}=\Phi_{\lambda} N_{1} \varphi, \quad \varphi_{n+1}=\Phi_{\lambda} N_{1} \varphi_{n}
$$


Используя последовательно леммы 2,3, получим следующие представления:

$$
\begin{gathered}
\varphi_{n}(x, \lambda)=K_{n} \varphi(x, \lambda)=\int_{0}^{x} K_{n}(x-t, t) \varphi(t, \lambda) d t, \quad n=1,2, \ldots, \\
K_{n}(x, \xi)=\int_{0}^{x} d t \int_{0}^{t} g(x-t, t-\tau) N_{n}(\tau, t-\tau) d \tau \\
+\int_{0}^{x} d t \int_{0}^{t} g(t-\tau, x-t) N_{n}(\tau, t-\tau+\xi) d \tau+\frac{1}{\alpha} \int_{0}^{\xi} N_{n}(x, t) d t \\
+\frac{1}{\alpha} \sum_{0<|j| \leqslant m}\left[\frac{\omega^{j}}{1-\omega^{j}} \int_{0}^{x} N_{n}\left(\tau, \xi+\frac{x-\tau}{1-\omega^{j}}\right) d \tau\right. \\
\left.\quad-\frac{1}{1-\omega^{j}} \int_{0}^{x} N_{n}\left(\tau, \frac{x-\tau}{1-\omega^{j}}\right) d \tau\right], \\
\quad N_{n+1}(x, \xi)=\int_{0}^{x} N_{1}(x-\tau, \xi+\tau) K_{n}(\tau, \xi) d \tau,
\end{gathered}
$$

$K_{n}$ - вольтерровы операторы класса $A$. Далее, из (42) следует

$$
\left|K_{n}(x, \xi)\right| \leqslant C \max \left\{\left|N_{n}(\tau, \zeta)\right|: \tau \in[0, x], \zeta \in \bar{D}_{1-\tau}\right\},
$$

где

$$
C=2 \int_{0}^{1} \int_{0}^{1}|g(x, t)| d t d x+\frac{4 m+1}{\alpha|1-\omega|} .
$$

Объединив (44) и (43), получим по индукции

$$
\left|N_{n}(x, \xi)\right| \leqslant C^{n-1} C_{1}^{n} \frac{x^{n-1}}{(n-1) !}, \quad\left|K_{n}(x, \xi)\right| \leqslant C^{n} C_{1}^{n} \frac{x^{n-1}}{(n-1) !},
$$

где $C_{1}=\max \left\{\left|N_{1}(x, \xi)\right|:\{(x, \xi) \in V\}\right.$. Определим

$$
K_{0}(x, \xi):=\sum_{n=1}^{\infty} K_{n}(x, \xi)
$$

В силу оценок (45) ряд в правой части (46) сходится абсолютно и равномерно на $V$, определяемая им функция $K_{0}(x, \xi)$ обладает теми же свойствами, что и $N(x, \xi)$. Из (40), (41), (46) следует

$$
y_{1}(x, \lambda)=\varphi(x, \lambda)+\int_{0}^{x} K_{0}(x-t, t) \varphi(t, \lambda) d t,
$$

откуда, в свою очередь, с учетом определения функции $y_{1}(x, \lambda)$ получается представление

$$
y(x, \lambda)=\varphi(x, \lambda)+K \varphi(x, \lambda)=\varphi(x, \lambda)+\int_{0}^{x} K(x-t, t) \varphi(t, \lambda) d t,
$$

откуда с учетом определения функций $y(x, \lambda), \varphi(x, \lambda)$ далее следует

$$
M^{n} 1=(E+K)\left(J^{\alpha}\right)^{n} 1, \quad M(E+K) \frac{x^{n \alpha}}{\Gamma(n \alpha+1)}=(E+K) J^{\alpha} \frac{x^{n \alpha}}{\Gamma(n \alpha+1)} .
$$

В силу полноты в пространствах $C[0,1], L_{p}[0,1]$ системы функций $\left\{x^{n \alpha} / \Gamma(n \alpha+1)\right\}_{n=0}^{\infty}$ заключаем, что в каждом из этих пространств выполнено равенство $(E+K) J^{\alpha}=$ $M(E+K)$. Теорема 1 доказана. 
ДоКАЗАТЕЛЬСТво теОРемЫ 2. Перепишем $\ell y$ в виде

$$
\ell y=D^{\alpha} y+\sum_{k=2}^{n} D^{n-k}\left(q_{k}(x) J^{\epsilon} y\right)+P J^{\epsilon} y,
$$

где

$$
q_{k}(x)=\sum_{j=2}^{k}(-1)^{(k-j)} C_{n-j}^{k-j} p_{j}^{(k-j)}(x) .
$$

Вычитая (7) из (5), получим

$$
D^{\alpha}(y-\tilde{y})-\lambda(y-\tilde{y})=-\sum_{k=2}^{n} D^{n-k}\left(q_{k}(x) J^{\epsilon} y\right)-P J^{\epsilon} y,
$$

откуда с учетом начальных условий (6), (8) имеем

$$
\begin{aligned}
y-\tilde{y} & =-\left(E-\lambda J^{\alpha}\right)^{-1} J^{\alpha}\left(\sum_{k=2}^{n} D^{n-k}\left(q_{k}(x) J^{\epsilon} y\right)-P J^{\epsilon} y\right) \\
& =-\Phi_{\lambda} J^{\alpha-1}\left(\sum_{k=2}^{n} D^{n-k}\left(q_{k}(x) J^{\epsilon} y\right)-P J^{\epsilon} y\right) .
\end{aligned}
$$

Окончательно, с учетом начальных условий (6) получается следующее интегральное уравнение относительно $y(x, \lambda)$ :

$$
y=\tilde{y}-\Phi_{\lambda} N J^{\epsilon} y
$$

где

$$
N=\sum_{k=2}^{n} J^{k-1-\epsilon} q_{k}(x) E+J^{\alpha-1} P .
$$

В силу аналитических свойств коэффициентов $p_{k}(\xi) N$-вольтерров оператор класса $A$. Решая уравнение (47) методом последовательных приближений, получим

$$
y=\tilde{y}+\sum_{j=1}^{\infty} y_{j}
$$

где

$$
y_{1}=-\Phi_{\lambda} N J^{\epsilon} \tilde{y}=-\Phi_{\lambda} N \varphi, \quad y_{j+1}=-\Phi_{\lambda} N J^{\epsilon} y_{j} .
$$

(Было учтено, что, как нетрудно видеть, $\varphi=J^{\epsilon} \tilde{y}$.)

Рассуждая, как при доказательстве теоремы 1 , получим для $y(x, \lambda)$ интегральное представление

$$
y=\tilde{y}+K_{0} \varphi,
$$

где $K_{0}$ - вольтерров оператор класса $A$. Воспользовавшись еще раз тем, что $\varphi=J^{\epsilon} \tilde{y}$, и положив $K=K_{0} J^{\epsilon}$, получим для $y(x, \lambda)$ требуемое представление (9). Теорема 2 доказана.

В заключение автор хотел бы выразить благодарность О.В. Седину за постановку задачи и полезные обсуждения. 


\section{СПИСОК ЦИТИРОВАННОЙ ЛИТЕРАТУРЫ}

[1] Сахнович Л.А. О приведении вольтерровых операторов к простейшему виду и обратньх задачах // Изв. АН СССР. Сер. матем. 1957. Т. 21. № 2. С. 235-262.

[2] Сахнович Л.А. Обратная задача для дифференциальных операторов порядка $n>2$ с аналитическими коэффициентами // Матем. сб. 1958. Т. 46. №1. С. 61-76.

[3] Хачатрян И.Г. Об операторах преобразования для дифференциальных уравнений высших порядков // Изв. АН АрмССР. Сер. матем. 1978. Т. 13. № 3. С. 215-238.

[4] Седин О.В. О подобии вольтеррова оператора $n$-й степени оператору интегрирования // Линейные операторы в функциональных пространствах. Грозный, 1989.

[5] Мацаев В. И. О существовании оператора преобразования для дифференциальных операторов высших порядков // Докл. АН СССР. 1960. Т. 130. № 3. С. 499-502.

[6] Маламуд М. М. Подобие вольтерровых операторов и смежные вопросы теории дифференциальных уравнений дробных порядков // Тр. ММО. 1994. Т. 55. С. 73-148.

[7] Диткин В. А., Прудников А. П. Операционное исчисление по двум переменным и его приложения. М.: Физматгиз, 1958.

Саратовский государственный университет им. Н. Г. Черньшевского

Поступило

E-mail: ignatievmu@info.sgu.ru

10.10 .2000 